\title{
Riesgos sociales en América Latina y políticas publicas: actores políticos y prácticas operativas
}

\author{
Riscos sociais na América Latina e políticas públicas: atores políticas e \\ práticas implementadas
}

\section{Risk social in Latin America and the social policy: political actors and operating practices}

\author{
Risques sociaux en Amérique Latine et politique publique: acteurs politiques et pratiques \\ de fonctionnement
}

\author{
Aloisio Ruscheinsky* \\ (aloisior@unisinos.br) \\ David Martínez** \\ (david.martinez@leon.uia.mx)
}

Recebido em 16/07/2012; revisado e aprovado em 18/10/2012; aceito em 14/03/2013

\begin{abstract}
Resumen: El artículo presenta consideraciones acerca de los mecanismos perversos de los riesgos sociales generados por las desigualdades, la explotación de un cuadro dramático de la injusticia y de las iniquidades. Los autores exponen algunos suportes teóricos de apoyo a la interpretación ampliada de las políticas sociales con dilatados riesgos sociales en el contexto del pluralismo de la pobreza extrema y bienestar en América Latina.

Palabras claves: Riesgos sociales. América Latina. Políticas sociales.

Resumo: O artigo apresenta considerações acerca dos mecanismos perversos dos riscos sociais gerados pelas desigualdades, revelando um quadro dramático de injustiça e de iniquidades. Expõe alguns suportes teóricos de apoio à interpretação ampliada das políticas sociais em face de amplos riscos sociais num contexto plural de pobreza extrema e bem-estar na América Latina.

Palavras-chaves: Riscos sociais. América Latina. Políticas sociais.

Abstract: The article presents considerations about the perverse mechanisms of social risks created by inequality, exploitation of a dramatic perspective of injustice and inequities. Brings some theoretical support to the interpretation that helps with a social policy analysis with dilated social risksin a context of extreme poverty pluralismoand welfarein Latin America. Key words: Social risks. Latin America. Social policy.

Résumé: Le article apporte considerátions sur les dispositives damnable des risques sociaux générée dans les inégalités, révélant un cadre dramatique de d'injustice et d'iniquités. Expose quelques nutriment théoriques de soutien au interprétation étendue de les politiques sociales face aux larges risques sociaux dans un contexte au pluriel de l'extrême pauvreté et de bien-être en Amérique latine.

Mots-clés: Risques sociaux. Amérique Latine. Politique publique.
\end{abstract}

\section{Introducción}

El texto que ahora se ofrece para su discusión tiene el propósito de presentar a los investigadores algunos aspectos sobre ejes ordenadores de la discusión conceptual y de las prácticas políticas calentadoras de una perspectiva de seguridad social a los ciudadanos de una nación. Los elementos para trabajar la discusión sobre política social comparada en América Latina se toman en cuenta la corriente de pensamiento que considera las contradicciones sociales y el conflicto entre actores sociales. La superación de la pobreza en circunstancias de extrema desigualdad depende del acceso a una gama de bienes materiales (dimensión económica) y también de bienes intangibles (de las relaciones de poder y culturales), siendo estos últimos denominados por algunos autores de capital cultural. En tal óptica se permitirá lograr un doble propósito: 1) compartir, discutir y sobre todo construir visiones, conceptos y enfoques homologables sobre el problema de la política social ante la persistente pobreza continental; 2) recortar y enfocar el objeto de investigación desde las opciones teórico/metodológicas combatibles con la realidad latinoamericana .

Las políticas sociales en América Latina han demostrado que su prácticas son un poderoso mecanismo para favorecer el desarrollo local, derrotando vulnerabilidades

\footnotetext{
*Universidade do Rio dos Sinos (Unisinos), São Leopoldo, RS, Brasil.

** Universidad Iberoamericana, León, México.
} 
múltiples. Las reflexiones teóricas presentadas en este artículo están directamente en el diálogo con los informes de las prácticas de investigación sobre el mismo tema en ocho países de América Latina.

En tal sentido un estudio particular de la naturaleza de las desigualdades históricas, cuya perspectiva desde nuestro punto de vista, es imperativa para tratar de entender por qué las políticas sociales y concretamente los programas sociales focales tienen impactos diferenciales en un país. Aquí es, desde nuestra perspectiva, en donde se genera el puente para hablar de prácticas políticas similares para combatir la pobreza en circunstancias de desempleo estructural. Sin lugar a dudas, las heterogeneidades visibilizadas llevan a hablar de las privaciones o de riesgos amplios de una parte de la población, pero entendemos a éstas en el marco de las diferencias y de los ejes que las producen dentro de un país. Las políticas sociales pretenden dar respuestas desde una perspectiva política a los problemas socio económicos en particular; pero la interrogación que se queda a responder es si las políticas sociales han implementado un juego de distribución que están cambiando las bases históricamente arraigadas de las desigualdades sociales.

El método de investigación fundamentalmente basado en las ciencias sociales para comprender la sociedad desigual tiene en cuenta, en general, las dimensiones políticas de la vida cotidiana y las condicionantes estructurales. Estas dimensiones no asumen acciones lineales, rompiendo con la división exacta de los espacios públicos y privados, pero tenían en cuenta el regresivo-progresivo en las encuestas y en la realidad histórica, sobre todo porque la historia y las prácticas políticas no siempre evolucionan para mejorar el ser humano. La perspectiva de los modelos y consecuencias de las políticas sociales es discutida en la parte segunda del presente texto, abordando el contexto de las políticas sociales del bienestar. En la tercera parte del artículo se intenta un repaso sobre los regímenes de bienestar con una mirada de debate en América Latina. Con la investigación se intenta enriquecer de forma crítica lo que sucede en múltiples partes del continente. La comparación de experiencias nacionales, como es razonable pensar, supone el diseño de líneas comunes para que puedan ser consistentemente confrontables (MANOW, 2007).

\section{El problema de una investigación social latinoamericana}

Las inquietudes académicas que confluyen en este texto constituyen interrogantes suscitadas sobre el debate alcanzado con respecto a la conceptualización, diseño, operación, evaluación y comparación de las políticas sociales en distintos países de América Latina y de una toma de distancia con respecto a la opción adoptada en la región que progresivamente ha mercantilizado y familiarizado la seguridad social y le ha asignado a la política social en su modalidad residual una cierta exclusividad en el "combate a la pobreza".

Consideramos que el análisis y la comparación de la trayectoria de la política social si se hace desde una mirada construida con conceptos que por su novedad y potencia han venido tomando forma a lo largo del la última década, pueden aportar elementos para replantear el papel de las distintas esferas productoras de bienestar y en ese sentido aportar algunas propuestas dirigidas a los gestores públicos y grupos de influencia en Latinoamérica para reenfocar las políticas sociales hasta ahora aplicadas.

El problema social que origina el interés por la investigación es la existencia de una incapacidad crónica para atender las demandas básicas de inmensas franjas de la población: "ni las recientes reformas sociales -caracterizadas por su énfasis en la focalización de los recursos públicos hacia la población pobre a fin de ofrecer servicios de carácter asistencial que mitiguen su condición" (MOLINA, 2006, p. vii) han dado los frutos debidos, pero reforzando una perspectiva populista. De la misma forma las reformas liberales precedentes, que abogaron por un universalismo de los servicios sociales apoyado por el Estado, en la práctica, separándose de sus objetivos originarios, no han dado los resultados pretendidos, porque descuidaron las restricciones políticas, institucionales, culturales y económicas.

La distribución de los servicios sociales dio lugar al incremento del gasto público y en los años ochenta ante una parte de la demanda no atendida hay quienes abogan por sustituir el modelo. En los años noventa, 
frente a la queda de credibilidad de la utopía universalista se comienza una reflexión sobre el carácter residual de las políticas sociales o el énfasis en las políticas focalizadas para erradicar el hambre y la exclusión en segmentos específicos.

Los avances en los niveles de vida de la población en América Latina han sido desiguales entre los países, aunque hay optimismo al respecto por parte de algunos organismos internacionales, la trayectoria en la primera década del siglo XXI ha sido inconsistente. En el documento Panorama Social de América Latina 2005 se señala que: "gracias al favorable desempeño económico alcanzado por América Latina en el último bienio y a los avances en la reducción de la pobreza logrados por algunos países, las tasas de pobreza e indigencia previstas para el año 2005 serían inferiores a $41 \%$ y $17 \%$, respectivamente, porcentajes que marcan los niveles más bajos de los últimos 25 años (CEPAL, 2005, p. 61). De esta manera, el contingente de los pobres podría declinar en una década a la cifra en que anteriormente de forma similar a la observada de millones que se encontrarían una vez en situación de pobreza extrema.

La realidad de millones de personas por debajo de un nivel de vida digno sin acceder al goce de los derechos humanos fundamentales que le son inherentes, se encuentran frente a una elite que acumula riqueza y poder, que aplicó en Latinoamérica las reformas neoliberales, con inserción subordinada a la economía global, apoyando la retirada del Estado, naturaliza la expansión de la miseria, del desempleo y del trabajo informal. En esta circunstancia se "han conducido a la sustitución de políticas de raigambre universalista (por poco desarrolladas que estuvieran) por otras de tipo focalizado que han impactado de forma negativa sobre la ciudadanía social" (ADELANTADO, 2005, p. 29).

Para convertir el problema social expuesto en este panorama de la desigualdad en América Latina a un problema de investigación, resulta crucial contar con un aparato conceptual sólido que permita aportar un punto de vista novedoso al debate que universidades, centros de investigación y agencias internacionales vienen impulsando. Es pertinente indagar sobre los paradigmas del bienestar sustentables, los regímenes de bie- nestar que operan en la realidad (MANOW, 2007), el proceso de institucionalización con que se intenciona la producción de bienestar y las estrategias programáticas que se han aplicado en las diferentes naciones de forma que se profundice la relación entre el bienestar de la población, la pobreza y la responsabilidad pública frente a la estructura de los riesgos sociales producidos. Esta afirmación adquiere relevancia si se considera que "en los países desarrollados, el estado de bienestar posee un núcleo institucional fuerte, sobresaliente y relativamente estable. Los cambios suceden alrededor de ese núcleo. No es éste el caso predominante en América Latina, donde no existe un conjunto de instituciones y programas sociales debidamente articulados por un conjunto estratégico de medios y metas sociales a largo plazo" (GALIANI, 2006, p. 12).

El objetivo explícito de abatir la desigualdad, como un asunto de índole ético y que por lo tanto garantice la sustentabilidad de sociedades latinoamericanas, no se reduce al buen funcionamiento de alguna de las tres estructuras básicas de la sociedad: la económica, la política o la social sino a un arreglo articulador de ellas. La noción de régimen de bienestar que más adelante se discutirá, junto con su correlato ideal, el de paradigma de bienestar, ayuda a este propósito pues provee de un enfoque vinculante entre las esferas del Estado, mercado y familia. Las modalidades que han permeado los distintos enfoques de la política social en nuestros países han contemplado de muy diferente modo el propósito de aumentar las capacidades de los grupos y personas y también en la reconstrucción del deteriorado tejido social que ha sido vulnerado por diferentes factores, entre los que se encuentran la precarización del empleo, la delincuencia, el narcotráfico y la violencia.

\section{El conflicto social y político de las prácti- cas políticas operativas}

Existe una extensa y compleja discusión sobre las escuelas de pensamiento que se encuentran en la base de los estudios de la política social y la pobreza, no sólo en América Latina. En la elección construcción de un posicionamiento se lleva en consideración que "la política social es un campo de batalla, una zona donde compiten opciones prácticas, 
ideologías y enfoques teóricos distintos" (BARBA, 2006, p. 29).

Para la investigación que nos proponemos impulsar, se evita de entrada la elección de alguna corriente que tenga un calificativo elaborado de antemano (crítica, instrumental, social demócrata, conservadora o administrativa, entre otras) y a partir de ella derivar las tesis fundamentales de la articulación. En lugar de eso se proponen tres ejes de discusión que caractericen los puntos álgidos del debate y en una construcción que tendrá que abrir el espacio para señalar acuerdos, desacuerdos y ajustes.

Una elección que se debe explicitar y que proviene de cierto rigor metodológico es que nuestro objeto de investigación tiene en su núcleo central a la política social, en cuyo prospecto vamos a tratar de correlacionar las formas de protección o bienestar social y los caminos de la ciudadanía en consonancia con da literatura sobre el fenómeno en curso en los países de Latinoamérica (DRAIBE; RIESCO, 2006; ANDRENACCI, 2006). En efecto, nos interesa traer elementos para referir a las distintas experiencias de política social y desde ahí observar su relación con el combate a la pobreza. Por lo tanto la pobreza tendría el carácter de variable dependiente en el caso de que siguiéramos una investigación hipotética deductiva, o para ser más claros en una visión constructivista, las preguntas de investigación pondrán énfasis en la política social como núcleo central articulador.

Por lo tanto nos referimos ahora a tres ejes organizadores para la discusión: la política social y el bienestar (paradigma y régimen) en la historia contemporánea (BOSCHETTI et al., 2008); la política social en su relación con el abatimiento de la desigualdad y la pobreza; la política social en el marco de la construcción de la ciudadanía. El primer eje es considerado como el central pues contiene definiciones de fondo que serán profundizados en los dos ejes restantes. Vale la pena subrayar que sólo son ordenadores de la discusión, es decir, elementos aglutinadores y puertas de entrada que dan pie al tratamiento de otros conceptos.

Del conjunto de aproximaciones teóricas tomaremos a Gosta Esping-Andersen (2000; 2009), también los aportes realizados por Barba (2000; 2006) y Filgueira et al. (2006), así como los apuntes de José Adelantado
(2000). Aunque estos autores tienen diferencias en el tratamiento de este primer eje de comprensión de las políticas de combate a la pobreza, en el desarrollo de este apartado se encontrarán aspectos comunes y complementarios en sus reflexiones, muy útiles en la comprensión del concepto de política social ubicado en una discusión más amplia del régimen del bienestar. Una primera perspectiva general en la que los autores coinciden sería la siguiente: 1) La necesidad de impulsar una reflexión crítica sobre el papel del mercado en el problema del bienestar. Los autores coinciden en la imposibilidad de encontrar o suponer un mercado regulador o de subordinar las decisiones sociales a un criterio mercantilizante, pero también se reconoce la imposibilidad de hablar del bienestar social sin incorporar esta dimensión.2) La impertinencia de que la política social pueda estar direccionada exclusivamente al combate de la pobreza. Sus objetivos profundos se encuentran en otras esferas: el logro de la restitución del tejido social, la disminución de la desigualdad, el aumento de capacidades de la población y la búsqueda del bienestar. 3) La existencia de esferas que son responsables del bienestar social: mercado, familia, estado y sociedad civil. Esta última es introducida, con fuerza, por Adelantado. 4) Los riesgos (objeto de la política social) de clase están asociados a los problemas de pobreza, y la atención a los sectores excluidos tiene su fundamento en un enfoque basado en derechos ciudadanos y no en la concesión asistencial y voluntarista de los gobiernos. 5) Las formas de enfrentar los riegos sociales son propios de cada país y no existen formas simples de importar o exportar esquemas sociales que no estén enraizados en la propia historia local. 6) La aceptación de las "nuevas" dinámicas que se generan en los últimos años, tales como el enfoque de género y los problemas del medio ambiente, como elementos constitutivos de una buena política social. 7) La política social no es un costo enviado a un fondo perdido, sino que colabora activamente en la dinamización económica de una sociedad.

Estos desconciertos introducen la búsqueda de algunas de las directrices teóricas de referencia, particularmente las relacionadas con los regímenes de bienestar, el universalismo básico, las fuentes del bienestar, 
la familiarización, la mercantilización y la relación de la política social con el bienestar (FRANZONI, 2007). De diversos modos se ha tratado de definir el concepto de bienestar, ya sea como un estado al que se llega a través de la posesión de ciertos bienes sociales, como es la comprensión de Lechner (2002) una mera resultante subjetiva de grupos y personas o incluso anteponiendo al bienestar el bien-ser, para connotar una subjetividad o una dimensión espiritual trascendente del ser humano que no se agota en los logros materiales.

Al modo de cómo Amartya Sen (1998; 2000) conceptualiza el desarrollo humano, se entenderá el bienestar como una capacidad, pero no como un estado de factor consumado. La capacidad sirve especialmente para el manejo de la incertidumbre en la contemporaneidad que se historiza de formas distintas (FRANZONI, 2008): la enfermedad, la vejez, la discapacidad, el ambiente, el extranjero, la discriminación, el desempleo, la etnia, el divorcio, la muerte. Todas estas, entre otras, son situaciones que están sujetas a la incertidumbre vital, es decir, son ejemplos de riesgos de múltiples órdenes en una sociedad desigual e inicua, que requieren una amplia democratización (MOLINO, 2005).

Si se afirma que el bienestar es una capacidad para el manejo de los riesgos sociales, se sigue que en contextos latinoamericanos tal capacidad como otros procesos y bienes sociales está distribuida inequitativamente entre la población como producto de las inserciones distintas en la estructura social. Con este efecto, los riesgos se presentan menores como producto de la mayor o menor capacidad de los individuos pero son consecuencia de arreglos sociales específicos que dan lugar a desigualdades y dominaciones étnicas, políticas, económicas y de género, entre otras. Para aprehender estas desigualdades es necesario contar con un nivel intermedio de análisis, que permita ubicar las desigualdades e inequidades, la dominación y opresión en los contextos específicos.

El concepto de régimen de bienestar se refiere a la "manera combinada e interdependiente como el bienestar es producido y asignado por el Estado, el mercado y la familia" (ESPING-ANDERSEN, 2000, p. 34), es decir el efecto de las esferas dichas en las condiciones sociales de la población. La particular forma de articularse de esta tríada produce regímenes de bienestar diferentes. En el nivel macro, la producción de bienestar de cualquiera de los tres se encuentra relacionada con lo que ocurre con los otros dos. Y en el nivel micro, el bienestar de los individuos depende de cómo logren agrupar los inputs procedentes de los tres.

\section{Política social y riesgos sociales en una trayectoria}

Desde la perspectiva del igualitarismo y de la construcción de una cultura democrática, el principal objetivo de la política social es asegurar protección a la población contra los riesgos sociales, en este sentido se entenderá a la política social, en primera instancia y como una definición que contextualiza otras más operativas, como la gestión pública de los riesgos sociales. Los conflictos entre los actores sociales caracteriza la construcción democrática en América Latina (DAGNINO et al., 2006), aunque tenemos una sociedad que es profundamente plural y con inmensas desigualdades históricas.

Algunos riesgos son democráticos, en la medida misma en que se propagan en las redes sociales o debido a que afectan a todos los ciudadanos como la vejez, la contaminación ambiental, la alimentación, la enfermedad como señala Esping Andersen (2009), pero otros, como la pobreza, la inseguridad y el desempleo, se ligan estrechamente a la estratificación social. Así se considera la creciente heterogeneidad de la trayectoria de la vida, con las oportunidades y las capacidades más o menos iguales. En el contexto latinoamericana debe matizarse esta afirmación pues como fenómeno emergente se presenta el envejecimiento de la población, pero en conjunto de los contextos de pobreza, desempleo y sub empleo. Cuanto más generalizados son los riesgos sociales más probable resulta que la familia y el mercado fallen, volviéndose incapaces para absorber los riesgos de manera suficiente. La necesidad del Estado de bienestar resulta mucho mayor cuando consideramos el funcionamiento del mercado, con sus fallas y con sus éxitos. Si el fallo consiste únicamente en que se excluyen los riesgos inaceptables, una solución sería un estado de bienestar de carácter residual. A priori no hay ninguna 
razón por la cual la política social debería preocuparse por los riesgos aceptables.

Los riegos sociales pueden ser aleatorios, pero la mayoría se pueden dar con regularidad sociológica y se pueden dividir en tres riesgos (ESPING-ANDERSEN, 2000, p. 193-195): Riesgos de clase; de la trayectoria vital; Riesgos intergeneracionales. Los riesgos de clase y los intergeneracionales requieren de una solución en forma del Estado de bienestar. Allí, donde el Estado absorbe los riesgos, la satisfacción de la necesidad resulta tanto "desfamiliarizada" es decir, extraída de la familia como "desmercatilizada", extraída del mercado.

Los riesgos de trayectoria vital son los riegos sociales se distribuyen de manera desigual a lo largo de la trayectoria vital, así la pobreza se concentra especialmente en la infancia y en la vejez debido a la disminución de ingresos. El ciclo vital de la pobreza se encuentra asociado a la falta de correspondencia entre las necesidades y los ingresos específicos: las familias jóvenes tienen necesidades costosas y bajos ingresos; luego, los ingresos aumentan cuando los hijos se han marchado y después se reducen, drásticamente, en la vejez. Tradicionalmente, es en la familia donde se comparten los riesgos de la trayectoria vital. El contrato intergeneracional implicaba que los jóvenes atendían a los viejos a cambio de la transferencia de riqueza de éstos a aquellos. Si los ingresos permiten un ahorro en la etapa de la juventud, por vía del mercado también se ayuda a gestionar riesgos por vía de los seguros. Los nacientes rasgos de la sociedad pos industrial con su incremento de la inestabilidad familiar, su desempleo generalizado y su mayor inseguridad profesional, sugieren que los riesgos asociados a la trayectoria vital tienden a agruparse ahora en la juventud y los inicios de la vida adulta.

Los riesgos de clase y de la trayectoria vital demandan básicamente una cuestión de protección social y de seguridad de los ingresos, y el principal tipo de igualdad que implican es el de la universalización de los derechos. Los riesgos intergeneracionales exigen un compromiso con el igualitarismo distinto, básicamente en forma de políticas de igualdad de oportunidades.

La igualdad de oportunidades se puede interpretar desde una óptica minimalista (ase- gurar un acceso igualitario al capital humano eliminaría las desigualdades heredadas) y maximalista (introducen nuevos programas de discriminación positiva, el principio subyacente es que la desventaja pude provenir de múltiples fuentes y que todos los grupos deben hacer frente a diferentes riesgos). Hay que resolver no el problema de algunas persistentes desigualdades aisladas sino la reproducción sistemática de las desigualdades. El ataque a ésta debía de implicar todas las capacidades humanas en general: se trata de poner a disposición de la gente todo el conjunto de recursos socialmente disponibles.

La polémica y muy contemporánea cuestión de la desmercantilización (sacar del mercado un bien y convertirlo en universal) del Estado de bienestar, así como el debate de los modelos de solidaridad, se hallan unidos a la gestión de los riesgos sociales. La desmercantilización aspira a captar el grado en que los Estados de bienestar debilitan el nexo monetario al garantizar unos derechos independientes de la participación en el mercado, justificación por qué el principio de la desmercantilización de las políticas sociales combina con la defensa de los derechos humanos (ZIMMERMANN; SILVA, 2009). Para muchas mujeres el concepto de desmercantilización no resulta operante pues están incorporadas parcialmente al mercado laboral. El trabajo de las mujeres marca la expectativa de la reducción de su dependencia económica de otros ciudadanos y de los dilemas o (in)capacidades del Estado republicano (ANDRENACCI, 2006).

Para un conservador, una política "pro familia" implica un intento de restaurar los valores familiares y para otro significa un Estado de bienestar atento con las mujeres: una política activa orientada a reducir las cargas relativas al cuidado de la familia, o de los niños. La independencia femenina necesita desfamiliarizar las obligaciones relativas al bienestar. De ahí que el término de familiarización no incluye una postura en contra de la familia, sino está dirigido a captar el grado en que los riesgos sociales se van dirigiendo hacia la responsabilidad de la esfera doméstica. Familiarismo comprende aquel Estado de bienestar que asigna un máximo de obligaciones de bienestar a la unidad familiar (ANDRADE, 2009). Desfamiliarización comprende 
aquellas políticas que reducen la dependencia individual de la familia, que maximizan la disponibilidad de los recursos económicos del individuo, independientemente de las reciprocidades familiares o conyugales.

En el análisis de las políticas sociales que han operado en cualquier nivel espacial, tanto el papel asignado al mercado como a la familia son indispensables, si acaso se desea observar con cierto rigor los efectos y los supuestos bajo los cuales se estructura el aparato público destinado al bienestar social. Junto con avances en los promedios generales de matriculación escolar y esperanza de vida -por cierto a un nivel menor de lo que demandan las condiciones de existencia de la población empobrecida- coexisten problemas graves como la discriminación de indígenas, mujeres y campesinos, la precarización del trabajo y los niveles de generación de riqueza deseados para un desarrollo sostenido y justo. Esto se denomina de avanzo en dirección al pasado (LAURELL, 2004). A las tradicionales formas de exclusión y discriminación se le agregan nuevos riesgos sociales relacionados con la trayectoria vital, el género, envejecimiento poblacional, la solidaridad y el tránsito generacional, la cohesión social, la pauperización de parte de los grupos de medianos ingresos y las formas de cómo se enfrentan los problemas de subsistencia. La declaratoria de Latinoamérica sobre la dificultad para cumplir las metas del milenio es una manifestación del problema, bien como requiere políticas públicas universales y eficaces ante el cambio en el marco de las transformaciones culturales y económicas (ARRAIGADA; ARANDA, 2004).

Una parte de esta discusión tiene que ver con concebir a los presupuestos sociales como gastos que, si bien es cierto impactan en el nivel de vida de la población, no producen ningún tipo de resultado en el desarrollo económico. Según Noguera (ADELANTADO, 2000, p. 495), así como existen barreras y discusiones en torno de una política social alternativa, también se encuentran barreras culturales e ideológicas. Hay una trayectoria de los discursos justificadores de recortes, austeridad y mercantilización de los contenidos de la política social (SADER, 2000). De este modo, se crea una cultura que presenta, como leyes inevitables, las tendencias de contención del gasto, privatización, mercado y familiarización del bienestar social.

\section{La reestructuración del bienestar o una política social operativa}

José Adelantado (2000; 2005), al igual que Esping-Andersen, previenen en contra de una cierta ceguera de las políticas sociales con respecto a los cambios que ocurren en las sociedades. Una de las más recurrentes es la inconsistencia de una política social que siga pensando en una familia con un breadwinner a la cabeza, cuando las transformaciones en la esfera doméstica han dado lugar a fenómenos como las familias post nucleares, las transformaciones que se desarrollan en torno del desarrollo sustentable y de las emergencias de problemas con la migración, el medio ambiente, las cuestiones étnicas y las minorías sexuales. Las políticas sociales intentan traer algunos cambios a las familias y los cambios en el ámbito familiar desafían la solicitud de políticas eficaces (ARRAIGADA; ARANDA, 2004)

En particular, hay la coincidencia de distintos autores en caracterizar los recientes años como parte de un ciclo de reestructuración del paradigma social. Adelantado (2000, p. 74) destaca cinco dimensiones del cambio, que se amplían en los nuevos escenarios, en los ámbitos de impactos políticos y los tipos de impactos predominantes: 1) La lógica de estratificación social que supone el paso de una sociedad de clases a una sociedad con multiplicidad de ejes de desigualdad y nuevos espacios de exclusión; 2) La transición desde el antiguo régimen fordista a una economía terciarizada, propia de la esfera económicolaboral; 3) La propia esfera familiar se envuelve en nuevas relaciones de género; 4) En el campo simbólico cultural, amplias capas sociales, valores e identidades desligadas de la lógica clasista, así como nuevas formas de intervención colectiva más allá de las organizaciones tradicionales como sindicatos y partidos, como democracia participativa y movimientos sociales, pero todas ellas de impacto transversal, de modo que atraviesan las políticas sociales; 5) Las nuevas dimensiones espacio temporales, articulaciones de tipo local-global, producto de una relación del Estado-Nación con el fenómeno de la 
globalización y con niveles de gobierno diferenciados al interior de cada país.

Para la investigación que ahora se presenta resulta relevante el aporte de Adelantado sobre todo en las dos últimas dimensiones pues la política social no se entiende como una propuesta lineal desde arriba, sino como producto de luchas y conflictos sociales; y el último punto abre la posibilidad de reinventar la política social desde ámbitos más reducidos, como puede ser el caso de una provincia, una región o un estado.

Párrafos arriba se asumió el concepto de política social propuesto por Gosta Esping-Andersen como la gestión pública de los riesgos sociales. Después de la presentación conceptual de varios teóricos existe la posibilidad de enriquecerla y hacerla más operativa para que también nos acerque a la construcción de ejes analíticos concretos. La forma de organización de la solidaridad en la lucha contra la pobreza y la malnutrición, a través de políticas sociales, se agrega a una ingeniería institucional, se reduce a una práctica de orientación con énfasis en la focalización y se desarrolla en compañía de las asociaciones con el tercer sector (TEIXEIRA, 2009).

La política social en relación al resto de las esferas no supone una relación lineal causa-efecto o mecánica sino que es recursiva. Esto implica que, a la vez que la política social es parte del todo social, no se comporta como una variable mecánica dependiente de cualquier otra esfera social: la política social es determinada pero también determina. En la formulación y aplicación de la política social participan un conjunto desigual de actores: gobiernos de los tres niveles, dependencias federales, sindicatos, iniciativa privada, universidades, sociedad civil, ayuntamientos, en fin, grupos con intereses variados que están empeñados en dirigir la cosa pública hacia direcciones diversas. A la vez de la concurrencia de factores políticos, también interacciona con las otras esferas de la estructura social como las estructuras económicas e ideológicoculturales.

De alguna manera se orientan políticas sociales, en Venezuela o en otras naciones latinoamericanas, con el objetivo de restablecer el equilibrio social incorporando a los sectores sociales que material y socialmente habían estado excluidas del acceso al bienestar y a la riqueza que se hay producido colectivamente, dice LaCruz (2006). Estas nuevas políticas distributivitas buscan garantizar el acceso mas equitativo a los servicios públicos, a la seguridad alimentar, fundándose en el reconocimiento integral de los derechos sociales, económicos y políticos. Así la universalidad se entiende como la garantía de los derechos sociales establecidos en la Constitución, mediante la aplicación de políticas progresivas orientadas al disfrute de una vida con salud plena.

El problema de las desigualdades resulta complejo, para adelante la pobreza material y en términos de bienestar social. "Una definición de política social que nos permita relacionarla con la estructura social debería de contemplar esa dualidad constituyente y modulativa a la vez, en tanto que organiza una parte de la distribución de las cargas, beneficios y poder entre grupos y categorías de personas" (ADELANTADO en ARTEAGA, 2001, p. 67), y expresa lo que podría ser nuestra definición operativa :"La política social se articula en un conjunto de decisiones y actuaciones público-administrativas, generadas en la esfera estatal, que inciden directamente y de formas diversas sobre la organización y distribución de los recursos de bienestar, y lo hace mediante regulaciones, servicios y transferencias" (ADELANTADO en ARTEAGA, 2001, p. 67).

Inspirado en los trabajos de Samuel Lichtensztejn en la reflexión sobre las alternativas del desarrollo, Aguirre y Roman (2002, p. 5) presentan una definición similar para la política económica. Si hacemos una traducción libre, la de política social sería: el proceso mediante el cual un órgano de decisión (gobierno, en este caso) utiliza deliberadamente un conjunto de instrumentos para alcanzar ciertos objetivos relacionados con el bienestar social, que repercuten directa o indirectamente sobre diversos destinatarios sociales e implica cuatro elementos fundamentales: la autoridad, los fines, metas u objetivos, los instrumentos o mecanismos y los destinatarios.

\section{Los sustentáculos del universalismo bá- sico o política operativa}

Por su parte Barba (2006), hace una extensa argumentación sobre la valía de distin- 
guir el régimen y el paradigma de bienestar. El paradigma es un tipo ideal de bienestar que otros autores llamarían modelo - contiene las teorías y los conceptos que se construyen para definir, explicar y medir el bienestar social, "los instrumentos públicos que se diseñan y utilizan para alcanzarlo, así como el papel que les atribuye al mercado, al Estado y a la familia" (BARBA, 2006, p. 95). El régimen de bienestar es un arreglo realmente existente donde confluyen distintos paradigmas, es entendido "como un arreglo de situaciones específicas, donde diversos paradigmas se articulan y adquieren modalidades particulares, relacionadas con la historia privativa de la política social en cada lugar" (BARBA, 2006, p. 97).

Uno de los paradigmas que han entrado recientemente como propuesta a los problemas de la política social en América Latina es el de universalismo básico y conviene precisarlo pues para nuestros propósitos de investigación puede ser no sólo una alternativa de práctica política sino una forma estimulante de entrar al análisis de los problemas del bienestar social en la Latinoamérica. Párrafos arriba se señaló que Molina (2006), sostiene que ni las recientes reformas sociales caracterizadas por los afanes focalizadores ni las reformas precedentes que abogaron por un universalismo de los servicios sociales han dado los propuestos resultados históricos.

Ante esta incapacidad, se abre paso la propuesta del universalismo básico que se ofrece como una nueva orientación para la política social del enfrentamiento de la pobreza, cuya perspectiva se ha elaborado ampliamente en la publicación "Universalismo básico: una nueva política social para América Latina". En el Molina (2006, p. vii) propone cuatro rasgos: "i) la cobertura universal de las prestaciones sociales esenciales y debe alcanzar a las categorías de la población definidas a partir de atributos que todas las personas cumplan; ii) las prestaciones (bienes, rentas o servicios) debe cumplir con estándares de calidad garantizados para todos; iii) la garantía del usufructo de las prestaciones debe estar garantizada por el Estado; iv) se parte de un conjunto delimitado de prestaciones esenciales que responderá a las particularidades y niveles de desarrollo de cada país". La propuesta del universalismo básico
[...] se apoya en un diagnóstico de los intentos y fracasos que la región ha evidenciado en materia de construcción de sistemas de políticas sociales efectivos, efi cientes, equitativos y sostenibles, y propone una alternativa con un norte y orientación simples: una cobertura universal de prestaciones y riesgos esenciales, que asegure el acceso a transferencias, servicios y productos que cumplan con estándares de calidad homogéneos otorgados sobre la base de los principios de ciudadanía, es decir, una cobertura que se distancie del principio de selección de benefi ciarios de servicios a través de la demostración de recursos y de necesidad que predomina en la región, y que busque que estos sean concebidos como derechos, pero también como generadores de deberes. (FILGUEIRA et al., 2006, p. 21).

El concepto básico del universalismo sugiere las políticas sociales en virtud de una orientación innovadora de la protección social en América Latina y se guía en ejes (Molina (2006): fortalecimiento de la ciudadanía y la igualdad como valor indispensable; construir el proceso de la política social articulada y completa de las intervenciones sociales; y el papel del Estado en garantizar las prestaciones básicas. La propuesta se basa en el discurso de la ciudadanía y la igualdad se define como el papel de las estatales que limitan su responsabilidad a las garantías de los derechos sociales, por lo que requiere una reflexión crítica ante una propuesta de universalismo básico (NARBONDO, 2006).

Un modelo de universalismo básico es compatible de acuerdo con Filgueira (2007) con las opciones para mejorar acceso y para enfrentar el desfinanciamiento de los sistemas de seguridad social y tiene tres rasgos: a) focalizar con programas especiales para sectores de bajos recursos; b) subsidiar el acceso a sistemas contributivos a sectores de bajos recursos; c) generar prestaciones universales de base no contributiva desde rentas generales. Algunas prestaciones de servicios públicos se universalizan y los demás se deponen a los sistemas privados.

Más allá del resumen de la idea del universalismo básico hay que interrogarse sobre el campo de los problemas de las políticas sociales para afirmar que esta propuesta se justifica en el actual contexto latinoamericano. En el planteamiento de los impulsores del uni- 
versalismo básico se dice que la arquitectura social históricamente conformada en América Latina se ha constituido de capas superpuestas que no ha sido pertinente en su articulación con las nuevas problemáticas producto de la inserción internacional del área y se caracteriza por (FILGUEIRA, 2007; MOLINA, 2006): 1) un corporativismo persistente combinado con la disolución de los viejos mecanismos de solidaridad; 2) la inexistencia de un pilar de prestaciones sociales no contributivo; 3 ) la falta de fuerza y dirección del mercado - y de sus marcos e instrumentos de regulación - para que amplios sectores de la población accedan a los bienes y servicios dirigidos a su plena inserción económica y social.

Como consecuencia coexisten en tres niveles claramente diferenciados de la población pobre objeto de programas focalizados y asistenciales; un piso cada vez menos sólido constituido de una frágil población integrada, un tercer piso de altos ingresos constituido por mercados seguros de salud, de jubilaciones y pensiones y educación. Para atender esta situación los autores señalan con mucha claridad algunos obstáculos y plantean como ejes de trabajo futuro las estrategias sectoriales, la viabilidad fiscal, política y de gestión y las necesarias traducciones y adaptaciones nacionales, contexto en el cual hay un retorno del ciudadano través de la lucha contra las desigualdades sociales.

Las debilidades de las democracias latinoamericanas se confirman en la institucionalización de la desigualdad y se confirma en las limitaciones de las políticas sociales focalizadas para romper la persitencia de la cuestión social. Adelantado y Scherer (2008, p. 117) señalan tres argumentos: "i) debilitan la ciudadanía social, ii) favorecen el clientelismo político y, iii) son asistenciales (no constituyen un derecho)". Más allá de esas limitaciones y la crítica académica hay las fuentes de nuevos actores y otras formas de acción colectiva con acceso a la información y la formación del capital humano que pueden conducir las democracias latinoamericanas a adoptar procesos políticos más inclusivos.

La evaluacion de la relacion entre las politicas sociales de contenido marcado por el universalismo o por el focalismo tiene controversia fuerte y así Grynspan (2006, p. 78) señala:
El llamado a una focalización que se contrapusiera a la universalidad de las políticas anteriores fue un llamado retórico, pero no necesariamente fue adoptado por todos los países en la misma magnitud. Actualmente, focalización y universalismo son políticas complementarias y no, como fue concebido en los años ochenta, políticas contrapuestas. La focalización está pensada hoy como una focalización que lleve a la inclusión de todos aquellos sectores vulnerables, de todos aquellos sectores excluidos, de todos aquellos sectores que están por debajo de la línea de pobreza, para que puedan ser incluidos en la corriente principal del desarrollo.

Otro debae, es que el diseño, concepción e implementación de políticas sociales con un sesgo de la desmercantilización constituye un pertinente mecanismo para que los ciudadanos tengan un nivel tolerable de bienestar y mediante la celebración de las cuestiones sociales y ambientales, cierta independencia frente a la lógica de competitividad mercantil. La desmercantilización se refiere al grado en que los individuos pueden mantener un nivel de vida, incluso en las condiciones en que se apartan, independiente de su voluntad y fuerza política, del mercado de trabajo. Como resultado, una característica innovadora del derecho social de la ciudadanía, dice respecto, según Sposati (2007) a la posibilidad de una orientación no mercantilista de las políticas sociales en una sociedad muy desigual.

Las políticas sociales como indican garantizan algún bienestar a los individuos que se quedan al margen en relación a los procesos de trabajo en una sociedad con el desempleo estructural. En esta visión se establece un proceso para ampliar la capacidad de los individuos para acceder a los recursos y oportunidades para tomar sus decisiones cotidianas. En la acción de los actores sociales y políticos no constan los fenómenos que por sí mismos son imponderables o insuperables, lo que despliega un vasto campo de posibilidades políticas y propuestas en el marco analítico del pensamiento social. Las diversas políticas de bienestar planeadas y empleadas a lo largo de las últimas dos décadas han incorporado algunas críticas relativas al papel que desempeñan los actores sociales, ahora tienen una perspectiva universal, ahora se les distingue por el foco de tipo compensatorio. 
En ambos casos se atestigua como en las diferentes estrategias nacionales de protección social a los empobrecidos se valora el papel de la acción desempeñado por las mujeres, lo que constituye un importante paso metodológico (CARDOSO, 1996) y una dirección política para llegar a la comprensión de un vacío cultural del funcionamiento de los diversos mecanismos de afrontamiento.

De cara a la investigación particular en los países latinoamericanos conviene considerar un par de notas que sostienen la propuesta del universalismo básico, con el objetivo de profundizar en los diagnósticos, no tanto para fundamentar la necesidad del cambio sino para generar elementos que permitan especificar las diferencias entre los países de la región y proporcione guías útiles para construir la viabilidad política, fiscal y de gestión para el universalismo básico.

\section{Consideraciones finales}

El enfoque de la comprensión penetrante de los mecanismos que forjan las políticas sociales incluye los fenómenos históricos en sí mismos dinámicos y cambiantes, debido a la coexistencia de los constreñimientos o las limitaciones macroeconómicas. Nos parece importante retomar luego algunos puntos que asoma el análisis de las Políticas Sociales para vislumbrar los riesgos y vulnerabilidades de los países de la región, en especial su finalidad explicitada por la gestión pública y su aplicación práctica por las políticas de combate a la pobreza. Si bien la interpretación en particular requiere algo empírico, metodológico y estadístico, es importante recordar que no carece de una perspectiva teórica clave pues son las estructuras de las sociedades nacionales que predisponen a un cierto tipo de riesgos y vulnerabilidad, que tampoco puede desentenderse - pues es la otra cara de la moneda - de la estructura que produce privilegios en sociedades periféricas o en desarrollo.

La exposición del Estado y de sus políticas sociales a las presiones de la sociedad no puede ser identificada de inmediato con las mejores referencias a las cuestiones sociales y la superación de los riesgos sociales. En la actualidad latinoamericana, el Estado y la habitual coalición pluriclasista apoyan la expansión de su legitimidad frente a los actores políticos y su dominio sobre la sociedad mediante las prácticas políticas con la incorporación de sectores excluidos.

Teniendo en cuenta la formación del pensamiento social y político y la moderna democracia de masas en el contexto de una economía capitalista en expansión se mantiene la posibilidad de mitificación de la cuestión social. El pragmatismo político en América Latina ha delineando su contorno en las prácticas políticas, a menudo discontinuas, y al revés de la agenda de los movimientos sociales o las demandas sociales, como peligrosa circunstancia a los fines de la democracia social.

La óptica de los actores sociales no se conjuga con el despojo político de la sociedad, con la justificación de falla en la teoría democrática o con la precedencia de la perspectiva administrativa en la primacía de la resolución de la cuestión social. De un modo general, cabe registrar el desafío de la dependencia - el ciudadano queda sumergido a la condición de cliente sobre la realización de las promesas de bien estar - delante de las políticas sociales, cuando se confiere al Estado de derecho a la conducción de los albos preferenciales de sus acciones para quitar de la deuda social generada por los riesgos sociales.

La perspectiva de los actores sociales no va acompañada de la sencillez política de la sociedad, con el argumento de insuficiencia en la teoría democrática o la primacía del punto de vista administrativo en la resolución de las cuestiones sociales. En general, cabe destacar el reto de la dependencia - el ciudadano se sumerge bajo la condición del cliente de las promesas de bienestar - en las políticas sociales, cuando los muertos de hambre entregan a la esfera del Estado a la realización de los objetivos históricos para suprimir la deuda social generada por los riesgos sociales.

Nuestra expectativa es asegurarnos de que en la interpretación de las políticas sociales estamos en el carril de ideas que nos llevan a entender que la producción de la inequidad es abordada desde la acción política y social. Hoy en día, la reflexión en el surgimiento de los derechos universales basadas en políticas sociales apoyados por el universalismo básico significa enfrentarse al dilema que plantea la cuestión de la alteridad, una vez que la trampa es tan deseado en este curso incluyen la 
pluralidad y la diferencia sin comulgar con la equidad, con la desigualdad social, sino para acercarse a la condición fundamental para la negociación de las demandas y conflictos entre los actores sociales, mientras que se centra en la equidad.

Esto se hace especialmente difícil debido a dos circunstancias actualmente en curso en la mayoría de las naciones de América Latina. La primera es que la inequidad percibida en el sistema de distribución de beneficios sociales, y la necesidad de corregir los desequilibrios existentes, se produce en un momento en que hay restricciones en el gasto público y existe la compresión de gastos de las empresas hacia una reducción global en el gasto social, que indica el movimiento inverso de la redistribución. El segundo, por lo tanto se hace difícil separar las proposiciones de los organismos internacionales de los cambios en los sistemas de distribución de beneficios en nombre de la justicia y el cumplimiento de las recomendaciones de esos mismos organismos de ajuste fiscal y la contención del gasto público.

\section{Referencias}

ADELANTADO, José (Coord.). Cambios en el estado de bienestar: politicas sociales y

desigualdades en España. Barcelona, España: Icaria Editorial/Universitat Autónoma de Barcelona, 2000.

ADELANTADO, José. Desigualdad, democracia y políticas sociales focalizadas. Barcelona, España: Universidad de Barcelona, 2005.

ADELANTADO, José; SCHERER, Elenise. Desigualdad, Democracia y Políticas Sociales Focalizadas en América Latina. Estado, gobierno, gestión pública. Revista Chilena de Administración Pública, Chile, n. 11, p. 117-134, Junio 2008.

AGUIRRE, Rodolfo; ROMÁN, Ignacio. México. Veinte años de política económica. Guadalajara México: ITESO, SIMORELOS, SAPRIN, 2002. CDRom.

ANDRADE, Fabricio F. Desfamiliarização das políticas sociais na América Latina: uma breve análise dos sistemas de proteção social na região. Barbarói, Santa Cruz do Sul, n. 31, p. 56-71, 2009.

ANDRENACCI, Luciano (Comp.). Problemas de política social en la Argentina contemporánea. Buenos Aires: UNGS-Editorial Prometeo, 2006.

ARRAIGADA, Irmã; ARANDA, Verônica (Org.). Cambio de las familias en el marco de las transformaciones globales: necesidad de políticas públicas eficaces. Santiago: CEPAL/ ONU, 2004.

ARTEAGA, Carlos; SOLÍS, Silvia. La política social en la transición. México: UNAM y Plaza y Valdés, 2001.
BARBA, Carlos. ¿Reducir la pobreza o construir ciudadanía para todos? América Latina: regímenes de bienestar en transición al iniciar el siglo XXI. Guadalajara: Universidad de Guadalajara. 2006

BOSCHETTI, I. et al. (Org.). Política Social no Capitalismo. Tendências contemporâneas. São Paulo: Cortez, 2008.

CARDOSO, José C. Fundamentos sociais das economias pós-industriais: uma resenha crítica de Esping-Andersen. BIB - Revista Brasileira de Informação Bibliográfica em Ciências Sociais, ANPOCS, São Paulo, n. 41, p. 71-92, 1996.

COMISSÃO ECONÔMICA PARA A AMÉRICA LATINA E O CARIBE - CEPAL. Panorama Social de América Latina 2005. Chile: CEPAL, ONU, 2005.

DAGNINO, Evelina. Los significados de ciudadanía en América Latina. En: CHAPARRO, Adolfo; GALINDO, Carolina; SALLENAVE, Ana M. Estado, democracia y populismo en América Latina. Bogotá: Ed. Universidad del Rosario, 2008.

DAGNINO, Evelina; OLVERA, Alberto; PAMFICI, Aldo (Coord.). La disputa por la construcción democrática en América Latina. México: FCE/CIESAS/Universidad Veracruzana, 2006.

DRAIBE, Sonia; RIESCO, Manuel. Estado de Bienestar, desarrollo económico y ciudadanía: algunas lecciones de la literatura contemporánea. Mexico: CEPAL, 2006.

ESPING-ANDERSEN, Gosta. Fundamentos sociales de las economías post industriales. Barcelona: Ariel, 2000.

Três lições sobre o Estado-providência. Lisboa: Campo da Comunicação, 2009.

FILGUEIRA, Fernando. Cohesión, riesgo y arquitectura de protección social en América Latina. Santiago de Chile: CEPAL, 2007.

FILGUEIRA, Fernando y otros. Universalismo básico. Una alternativa posible y necesaria para mejorar las condiciones de vida en América Latina. Documento de Trabajo I-57. Washington D.C.: BID/INDES, 2006. Disponible en: <http://www.ciesu.edu.uy>.

FLEURY, Sônia; MOLINA, Carlos G. Modelos de Proteccion Social. En: Diseño y gerencia de políticas y programas sociales. Washington, DC: Banco Interamericano de Desarrollo (BID)/Instituto Interamericano para el Desarrollo Social (INDES), 2002.

FRANZONI, Juliana M. Regímenes Del Bienestar en America Latina. Madri: Fundacion Carolina, 2007.

FRANZONI, Juliana M. Domesticar la incertidumbre en América Latina: Mercado laboral, política social y familias. Costa Rica: Universidad de Costa Rica, 2008.

GALIANI, Sebastián. Políticas sociales: instituciones, información y conocimiento. Chile: CEPAL, 2006.

GRYNSPAN, Rebecca. Universalismo básico y Estado: principios y desafíos. In: MOLINA, Carlos G. (Ed.). Universalismo básico. Una nueva política social para América Latina. México: BID/Ed Planeta, 2006. p. 75-82.

LACRUZ, Tito. Balance sociopolítico: una ciudadanía social inacabada. In: MAINGÓN, Thaís (Coord.). Balance y perspectivas de la politica social en Venezuela. Caracas: ILDIS-CENDES-UNFPA, 2006. p. 111-184. 
LAURELL, Asa C. (Org.). Estado e políticas sociais no neoliberalismo. São Paulo: Cortez, 2004.

LAVALLE, Adrián G. Cidadania, igualdade e diferença. Lua Nova, Cedec, São Paulo, n. 59, p.75-94, 2003.

LECHNER, Norbert. Las sombras del mañana. La Dimensión subjetiva de la política. Santiago: Lom, 2002.

MANOW, Philip. As vantagens institucionais comparativas dos regimes de Estado de bem-estar social e as novas coalizões na sua reforma. In: DELGADO, M.; PORTO, Lorena (Org.). O estado de bem-estar social no século XX. São Paulo: LTR, 2007. p.160-186.

MARTÍNEZ, David. Política Social y Pobreza en Guanajuato. Reconstrucción de una trayectoria útil para las entidades federativas. León: UIA, 2008.

MOLINA, Carlos G. (Ed.). Universalismo básico. Una nueva política social para América Latina. México: BID/ Editorial Planeta, 2006.

NARBONDO, Pedro. Critical reflections about basic universalism. Revista Uruguaya de Ciencia Política, Montevideo, n. 15, p. 151-172, 2006.

PEREIRA, Potyara A. Necessidades humanas: subsídios à critica dos mínimos sociais. 3. ed. São Paulo: Cortez, 2006.
SADER, Emir (Org.). Pós-liberalismo: as políticas sociais e o Estado democrático. São Paulo: Paz e Terra, 2000.

SEN, Amartya. Desarrollo y libertad. México: Editorial Planeta, 2000.

Teorías del desarrollo en el siglo XXI. In: EMERIJ, L.; NUÑEZ, Arco J. (Comp.). El desarrollo económico y social en los umbrales del siglo XXI. Washington: BID, 1998.

SPOSATI, Aldaíza. Modelo de proteção social não contributiva: concepções fundantes. In: ENAP: Curso de Formação de Multiplicadores. Brasília: Ipea, 2007. v. I, p. 2-31.

. En busca de un modelo social latinoamericano. In: MOLINA, Carlos G. (Ed.). Universalismo básico. Una nueva política social para América Latina. México: BID/ Editorial Planeta, 2006. p. 59-74.

TEIXEIRA, Zuleide A. Consenso progresista desde el Sur. En: QUIROGA, Yesko; CANZANI, Agustín; ENSIGNIA, Jaime (Org.). Consenso progresista: las políticas sociales de los gobiernos progresistas del Cono Sur. Equador, 2009. Disponible em: <http:/ / library.fes.de/ pdf-files/bueros/chile/06402.pdf $>$.

ZIMMERMANN, Clóvis R.; SILVA, Marina C. O princípio da desmercantilização nas políticas sociais. Cadernos CRH, Salvador, v. 22, n. 56, p. 345-358, 2009. 